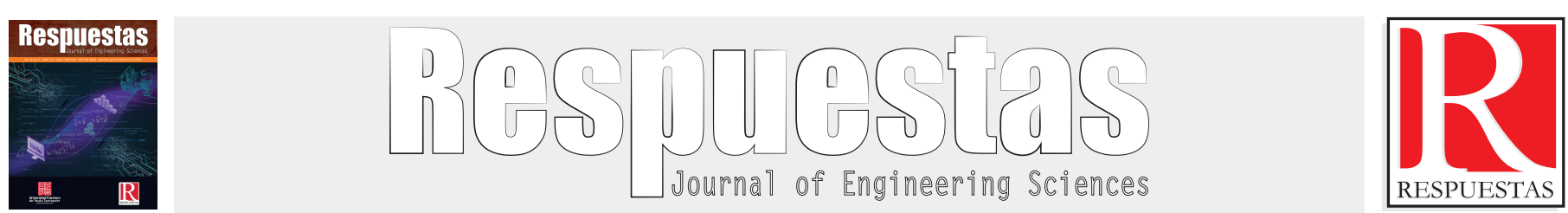

Artículo Original

https://doi.org/10.22463/0122820X.1491

\title{
Controlador de carga PWM eficiente y de bajo costo para sistemas fotovoltaicos autónomos
}

\author{
Low cost and efficient PWM charge controller for autonomous photovoltaic systems \\ Eduardo Andrés Luna-Paipa, Miguel Ángel Laguado-Serrano, Sergio Basilio Sepúlveda-Mora. \\ Ingeniero Electrónico, eduardoandreslp@ufps.edu.co, orcid.org/0000-0003-3518-3620,Universidad Francisco de Paula Santander, Cúcuta, Colombia. \\ ${ }^{b}$ Ingeniero Electrónico, miguelangells@ufps.edu.co,orcid.org/0000-0002-3505-4493,Universidad Francisco de Paula Santander, Cúcuta, Colombia. \\ ${ }^{c}$ Master of Science in Electrical and Computer Engineering, sergio.sepulveda@ufps.edu.co, orcid.org/0000-0002-1248-7616, Universidad Francisco de Paula Santander, Cúcuta, Colombia.
}

Cómo citar: E.A. Luna-Paipa, M.A. Laguado-Serrano y S.B. Sepúlveda-Mora, "Controlador de carga PWM eficiente y de bajo costo para sistemas fotovoltaicos autónomos", Respuestas, vol. 23, no. S1, pp. 6 - 13, 2018. https://doi.org/10.22463/0122820X.1491

Recibido: Febrero 07, 2018; Aprobado: Julio 16, 2018.

\begin{tabular}{|c|c|}
\hline & RESUMEN \\
\hline & $\begin{array}{l}\text { Los sistemas fotovoltaicos autónomos (SFA) se componen de un panel solar, una batería, una carga eléctrica y un controlador } \\
\text { de carga. En particular, el controlador de carga tiene como objetivo proteger la batería de sub-descargas y sobrecargas }\end{array}$ \\
\hline Controlador de carga & manteniendo un control constante en la energía entregada por el panel a la batería, así como también de la batería hacia la carga \\
\hline & conectada. En este artículo se describe el diseño e implementación de un controlador de carga utilizando modulación por ancho \\
\hline Microcontrolador & de pulso (PWM), tomando en cuenta un análisis de relación costo-beneficio para conseguir la mayor eficiencia posible a un \\
\hline Modulación por ancho de pulsos & precio competitivo en el ámbito comercial. Se desarrolló mediante un circuito de potencia utilizando transistores de efectos de \\
\hline Sistema Fotovoltaico Autónomo & $\begin{array}{l}\text { campo metal-óxido-semiconductor (MOSFET) de rápida conmutación; los MOSFET se activan a través de una señal PWM } \\
\text { transmitida por un micro-controlador PIC, que fue programado para variar el ciclo de trabajo de la señal PWM, dependiendo de } \\
\text { las variables que se miden en el SFA, como voltaje del panel y el estado de carga de la batería (SoC). El SoC se determina } \\
\text { mediante el método de conteo de Amperios-hora, midiendo las variables eléctricas utilizando divisores de voltaje y un sensor de } \\
\text { corriente de efecto Hall, los cuales envían la información al micro-controlador. Se implementó el controlador de carga utilizando } \\
\text { componentes de bajo costo y reduciendo la cantidad de sensores comúnmente usados sin afectar el funcionamiento del circuito, } \\
\text { además de obtener una eficiencia y un precio que compite con los controladores PWM existentes en el mercado. }\end{array}$ \\
\hline
\end{tabular}
además de obtener una eficiencia y un precio que compite con los controladores PWM existentes en el mercado.

\section{Keywords:}

Charge controller

Hall Effect

Micro-controller

Off-grid PV Systems

Pulse Width Modulation

\section{ABSTRACT}

An off-grid Photovoltaic (PV) system consists of a solar panel, a battery, an electrical load, and a charge controller. Particularly, the charge controller has the purpose of protecting the battery from overcharging and low discharging, controlling the energy delivered from the panel to the battery, and from the battery to the load. In this work, we described the design and implementation of a charge controller using pulse width modulation (PWM), taking into consideration an analysis of cost-benefit relation to obtain the highest efficiency at a competitive price in the market. The power electronics of the charge controller use Metal-Oxide-Semiconductor Field-Effect- Transistors (MOSFET); the MOSFETs are activated through a PWM signal transmitted by a PIC micro-controller that was programmed to change the duty cycle of the PWM signal according to the variables measured on the PV system such as solar panel voltage and the state of charge of the battery (SoC). The SoC is determined by the Ampere-hour counting method, measuring the electrical variables using voltage divisors and a Hall Effect current sensor; these devices send the information to the micro-controller. The charge controller was implemented using low cost components and reducing the number of sensor commonly used without affecting the operation of the circuit. Consequently, we developed a charge controller with an efficiency and price that competes with the current PWM controllers available in the market.

\section{Introducción}

Los sistemas fotovoltaicos se pueden dividir en dos clases, los sistemas conectados a la red eléctrica convencional [1] y los sistemas autónomos, siendo los segundos una de las alternativas actuales para combatir diversos problemas sociales producidos por la falta de energía eléctrica convencional [2] en poblaciones alejadas de la red eléctrica urbana donde la calidad de vida es baja debido a la falta de dispositivos eléctricos que facilitarían y mejorarían las condiciones de vida laboral [3] y cotidiana [4].

Los sistemas autónomos se componen de un panel solar, un controlador de carga [5], una batería [6] y una carga a alimentar, dependiendo de la carga a utilizar se requiere de un inversor.
En el caso de los controladores de carga, estos pueden funcionar mediante modulación por ancho de pulso (Pulse Width Modulation, PWM) [7] o con seguimiento del punto de máxima potencia (Maximum Power Point Tracking, MPPT) [8]; el objetivo principal de este trabajo es el diseño de un controlador de carga PWM que supere o mantenga la eficiencia de los controladores del mercado con un menor costo de adquisición.

El diseño del controlador consta de una etapa de control y una etapa de potencia. La etapa de control está basada en un microcontrolador programable PIC 16F877 que permite leer variables como voltaje y corriente, además de generar señales PWM cuyos ciclos de trabajo se modifican dependiendo del

*Autor de correspondencia. 
algoritmo diseñado utilizando el método de conteo Amperio-hora y la relación directa del voltaje de circuito abierto (Voltage Open Circuit, VOC) en la batería con el diagnóstico preciso del estado de carga de la misma [9]. Por otro lado, la etapa de potencia consiste en la implementación de transistores de unión bipolar (Bipolar Junction Transistor, BJT) y transistores de efecto de campo metal-óxido-semiconductor (Metal oxide - semiconductor Field-effect transistor, MOSFET) [10] para amplificar las señales PWM y generar conmutaciones entre las conexiones de panel, batería y carga.

Al implementar el controlador de carga PWM diseñado se realizaron mediciones de potencia entregada por el panel y potencia recibida por la batería en procedimiento de carga, para calcular una eficiencia promedio de $71.42 \%$. En el análisis de costos se tuvo en cuenta el precio de los elementos implementados en la realización del controlador, desde dispositivos electrónicos en el circuito hasta elementos usados en la adaptación estética, calculando un costo total de $\$ 59.300$.

\section{Materiales y métodos}

\section{Arquitectura del sistema fotovoltaico}

La arquitectura de un sistema fotovoltaico autónomo consiste principalmente en cuatro elementos: un arreglo de paneles solares, un controlador de carga, una batería, y una carga en DC. El panel solar usado en el sistema fotovoltaico implementado fue un panel solar policristalino de $30 \mathrm{~W}$, con un voltaje de máxima potencia de $18 \mathrm{~V}$ y una corriente de máxima potencia de 1.667 A. Se utilizó una batería de plomo ácido con un voltaje nominal de $12 \mathrm{~V}$ y una capacidad de $18 \mathrm{Ah}$, y como carga una lámpara Led de $12 \mathrm{~V}$ y $4 \mathrm{~W}$ de potencia.

\section{Diseño del circuito electrónico}

Selección de sensores: Las variables medidas por el controlador de carga PWM son: voltaje y corriente de batería, y voltaje de panel. Para medir los voltajes se optó por implementar dos divisores de tensión, usando solo dos resistores para cada uno. El divisor de tensión es una técnica de medición de bajo costo y de bajo consumo. La Figura 1 muestra uno de los divisores de tensión implementados, y el voltaje medido se calcula con (1).

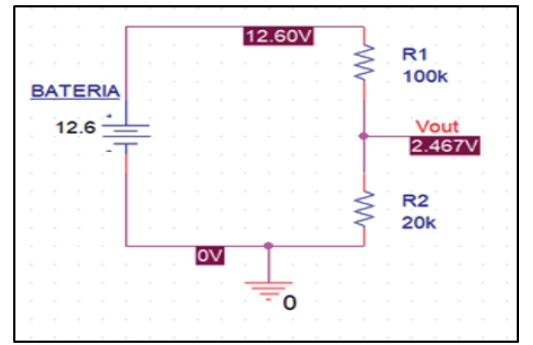

Figura 1. Divisor de tensión para medición de voltaje en la batería

\section{Donde:}

$$
\operatorname{Vin}=\frac{\operatorname{Vout}^{*}(R 2+R 1)}{R}(1)
$$

Vin es Voltaje de panel o batería (V)

Vout es Voltaje leido por el microcontrolador (V)

En cuanto a la medición de corriente por parte del controlador se decidió usar solo un sensor conectado al terminal positivo de la batería y de esta manera medir la corriente que entrega la batería cuando se descarga, así como también la corriente que recibe cuando se carga recibiendo potencia por parte del panel. Para reducir costos, la medición de la corriente de salida del panel no se realizó, pero esto no afecta la funcionalidad del controlador debido a que el voltaje del panel se mide de manera constante, y la caracterización realizada sobre el panel permitió determinar que $14 \mathrm{~V}$ medidos en el panel es suficientemente alto para cargar la batería.

El sensor elegido teniendo en cuenta costos, precisión e implementación sencilla es el circuito integrado ACS712, el cuál funciona basado en el efecto Hall, detectando el campo magnético generado por inducción de la corriente que circula por la línea medida, el sensor crea un voltaje eléctrico proporcional en respuesta a la corriente medida.

El módulo sensor de corriente ACS712 para Arduino está configurado y tiene conectado todo lo necesario para el funcionamiento del sensor como se muestra en la Figura 2; cuenta con dos terminales para conectar en serie la línea a la que se desea medir corriente, además de tres pines, dos para su polarización a $5 \mathrm{~V}$ y un tercer pin como salida analógica de voltaje; es éste módulo el que se implementó dentro de la circuitería del controlador PWM diseñado. El modelo específico implementado es el ACS712ELCTR-30A-T en su respectivo módulo tal como se muestra en la Figura 2, la conexión del sensor permitió medir tanto la corriente negativa entregada por la batería, como la corriente positiva recibida por la batería.

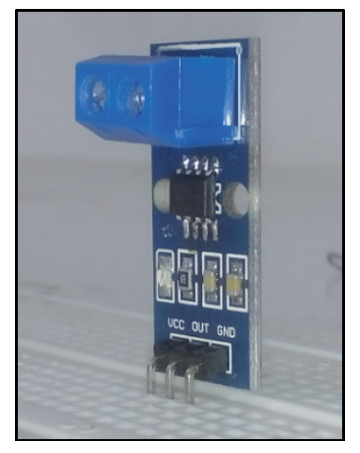

Figura 2. Sensor de corriente ACS712

Mediante (2) se describe el comportamiento lineal entre la corriente medida por el sensor y el valor de voltaje entregado por su salida analógica. Cuando la corriente medida es de $0 \mathrm{~A}$ la salida del sensor es de $2.5 \mathrm{~V}$, la sensibilidad en este modelo específico es de $66 \mathrm{mV} / \mathrm{A}$. 


$$
I=\frac{V-2.5}{66 \frac{\mathrm{mV}}{\mathrm{A}}}
$$

Donde:

$V$ es Voltaje de salida del sensor ( $V)$

I es Corriente medida por el sensor (A)

Diseño de la etapa de potencia: La conmutación realizada mediante las dos señales PWM se dan entre panel y batería, y entre batería y carga, el tiempo de conexión y desconexión depende del ciclo de trabajo de la señal PWM, el cuál varía dependiendo de la lógica en el algoritmo a programar en el microcontrolador y en respuesta a las variables de voltaje y corriente medidas por los sensores. Es necesario implementar interruptores electrónicos de alta potencia, por ello se optó por utilizar un MOSFET. Los transistores MOSFET son ideales para implementar conmutaciones a altas frecuencias ya que no es audible su cambio de estado a alta velocidad, por lo cual se pudo determinar una frecuencia de $20 \mathrm{kHz}$ para las señales PWM a implementar. Se seleccionó un transistor MOSFET tipo p IRF9540 con voltaje Drain-Source máximo de -100 V y corriente de drenado máxima de $-13 \mathrm{~A}$.

Las salidas PWM del microcontrolador tienen magnitud de voltaje máximo de $5 \mathrm{~V}$, valor de voltaje de un nivel lógico alto. Sin embargo, para llevar los MOSFET a conducción es necesario un voltaje mayor, es por ello que fue necesario implementar una etapa de amplificación utilizando transistores BJT.

Con el objetivo de obtener un voltaje mínimo de -6V en VGS para el disparo del MOSFET, se realizó el diseño circuital mostrado en la Figura 3, donde la fuente de $5 \mathrm{~V}$ simula la salida PWM del microcontrolador y la fuente de voltaje para la alimentación de RC son el panel o la batería. Se hallaron los valores de las resistencias necesarias mediante (3), (4) y (5) teniendo en cuenta que el Beta de los transistores disponibles para implementar era de 210 y 200.

$V R C=R C^{*} I c$

DconderC $=\frac{V R C}{500 \Omega}=12 \mathrm{~mA}$

$V R C$ es voltaje en la resistencia del colector del transistor $(V)$

$R C$ es resistencia en el colector del transistor $(\Omega)$

Ic es corriente en el colector del transistor $(A)$

Tomando una resistencia RC de $500 \Omega$, y un voltaje VRC de $-6 \mathrm{~V}$, se obtiene:

$I c=I b * \beta \quad(4)$

$I b 1=\frac{I c}{210}=\frac{12 m A}{210}=57.14 \mu \mathrm{A}$

$I b 2=\frac{12 m A}{200}=60 \mu A$
Donde:

Ib es corriente en la base del transistor (A)

$B$ es Beta especifico del transistor.

Ib1 es corriente en la base del transistor con Beta igual a 210 (A)

Ib2 es corriente en la base del transistor con Beta igual a 200 (A)

$5 V-R B^{*} I b-0.7=0$

$R B 1=\frac{5 V-0.7 V}{I b 1}=\frac{4.3 \mathrm{~V}}{57.17 \mu \mathrm{A}}=72.21 \mathrm{~K} \Omega$

$R B 2=\frac{5 \mathrm{~V}-0.7 \mathrm{~V}}{\mathrm{Ib2}}=\frac{4.3 \mathrm{~V}}{60 \mu \mathrm{A}}=71.66 \mathrm{~K} \Omega$

Donde:

$R B 1$ es el resistor en la base del transistor con Beta igual a $210(\Omega)$ $R B 2$ es el resistor en la base del transistor con Beta igual a $200(\Omega)$ De acuerdo con los resultados obtenidos, se optó por una resistencia de $72 \mathrm{k} \Omega$ como $R B$ y $500 \Omega$ como $R C$ en ambos circuitos de amplificación.

La configuración para el transistor MOSFET IRF9540 se muestra en la Figura 3; para que este MOSFET tipo p se active debe aplicarse un voltaje negativo entre sus terminales Gate y Source, por esta razón se conectaron estos terminales a la resistencia de colector del transistor BJT de tal manera que el voltaje es de - $6 \mathrm{~V}$ en VGS cuando la salida del microcontrolador es de nivel alto. Entonces, se produce una señal PWM entre los terminales Source y Drain del MOSFET sincronizada con la señal PWM que genera la salida del microcontrolador. La conmutación se realiza entre los terminales positivos del panel, la batería y la carga, permitiendo de esta manera tener un terminal tierra común, interconectando los terminales negativos de los tres elementos principales en el sistema fotovoltaico.

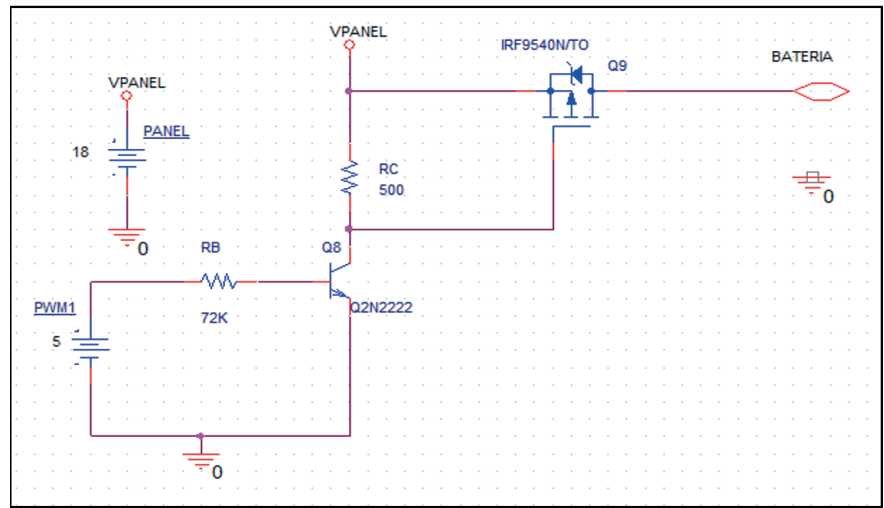

Figura 3. Configuración circuital para disparar la conmutación en el MOSFET

Diseño de la etapa de control y código programado al microcontrolador: Se seleccionó el PIC 16F877 ya que cuenta con 33 pines de entrada-salida donde 8 pueden ser utilizados como conversores A/D de 14 canales con resolución de 10 bits, con una frecuencia de operación máxima de $20 \mathrm{MHz}, 256$ bytes de memoria EEPROM, dos módulos CCP que sirven para generar la señal PWM, con una alimentación de máximo $5.5 \mathrm{~V}$ y una corriente de salida de $25 \mathrm{~mA}$ aproximadamente. 
El algoritmo desarrollado para el controlador PWM se basa entonces en tres funciones; la primera es el algoritmo principal donde se inicializan las variables, se ejecuta la medición de todos los sensores y se realiza la estimación inicial del estado de carga de la batería. Las otras funciones son las acciones a efectuar sobre los porcentajes de los ciclos de trabajo de las señal PWM dependiendo del estado de carga hallado en la primera función; estos porcentajes cambian mediante la medición del voltaje medido en la batería mientras está conectada al panel solar, y de la variación del porcentaje del estado de carga que es medido por el proceso del conteo de amperios hora [9]. Los diagramas de flujo del algoritmo se pueden ver en las Figuras 4, 5 y 6 , y el código fuente del controlador se encuentra disponible en el siguiente repositorio de GitHub: https://github.com/MiguelLaguado/ControladorDeCarga.

\section{Resultados}

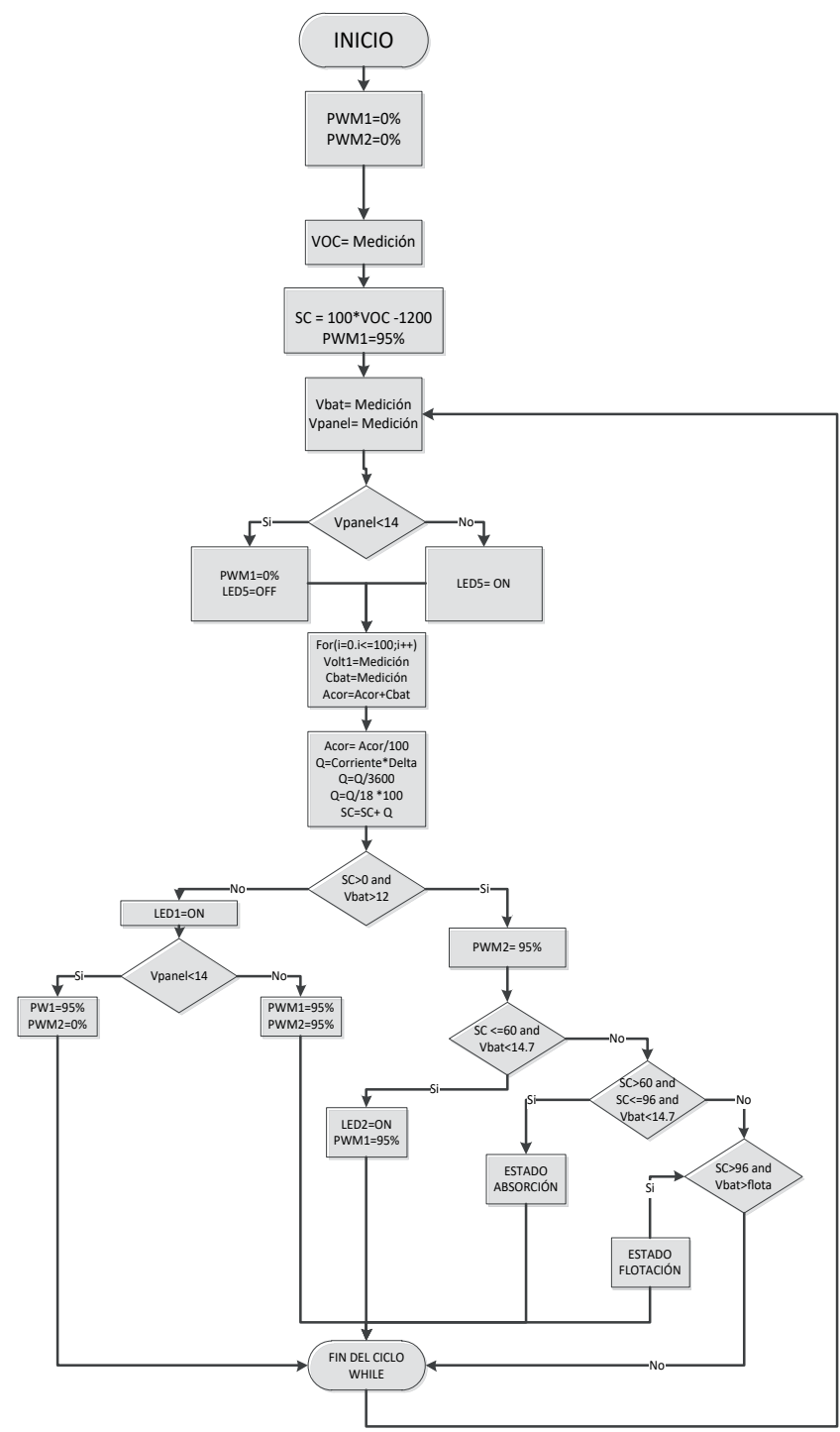

Figura 4. Algoritmo de carga del controlador de carga PWM

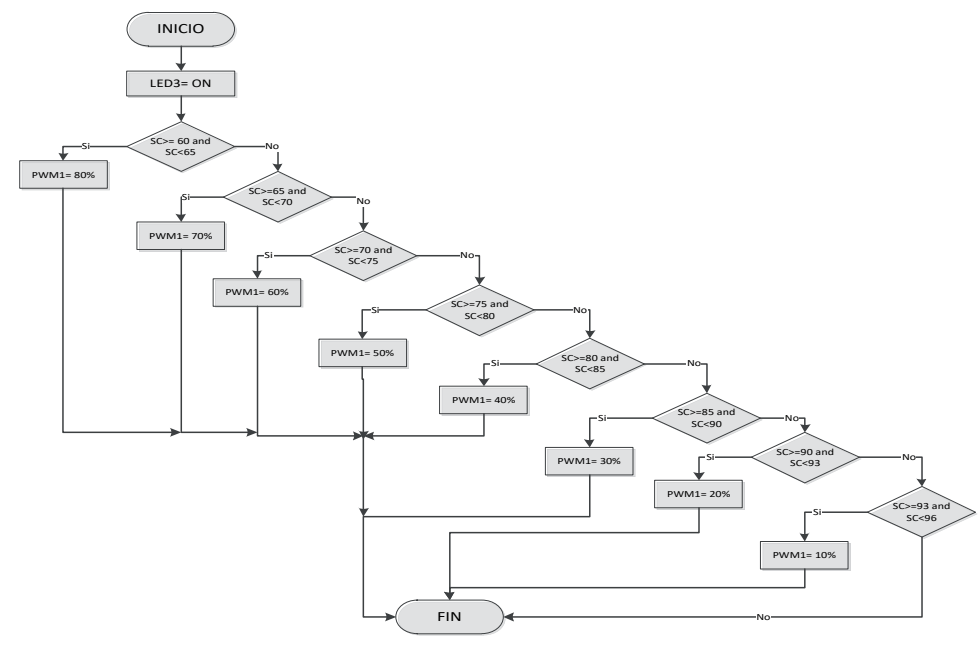

Figura 5. Diagrama de flujo del estado de absorción.

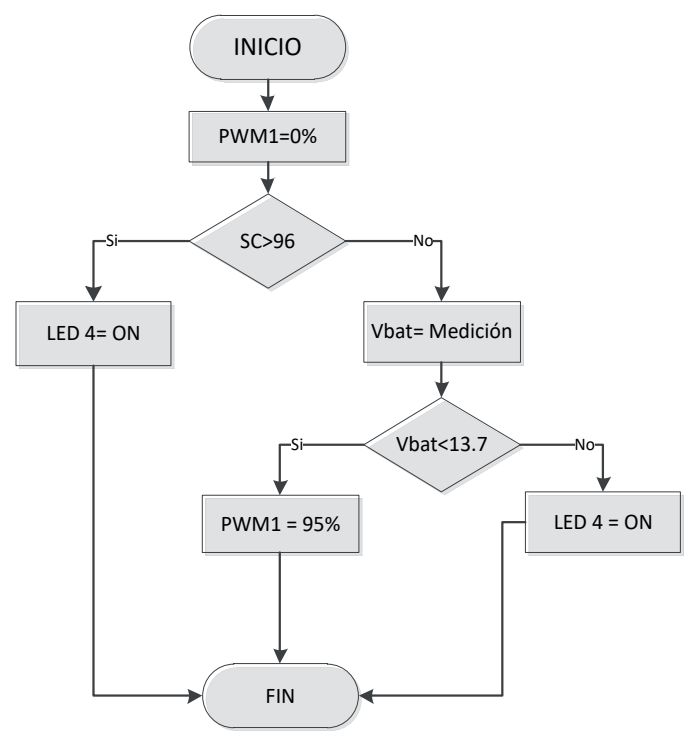

Figura 6. Diagrama de flujo del estado de flotación.

A continuación se muestran los resultados de la implementación del controlador de carga PWM en circuito impreso (Printed Circuit Board, PCB); se realizaron mediciones de potencia entregada por el panel y potencia recibida por la batería con el objetivo de calcular la eficiencia del controlador en el proceso de carga de la batería. Se implementaron conexiones experimentales donde se midieron de forma directa los voltajes en la batería y en el panel implementando voltímetros, y se calcularon corrientes a partir de voltajes medidos en resistencias de potencia de $1 \Omega$ conectadas en serie, tanto en la batería como en el panel mientras se dejaba en funcionamiento el controlador durante tiempos prolongados para comprobar los cambios de los estados de carga y probar su comportamiento cuando se somete a las condiciones normales de operación a los que un regulador de carga debe trabajar. Además, se midió la radiación solar incidente sobre el panel, implementando para ello un piranómetro del cual se medía su salida de voltaje y este valor se convertía a $\mathrm{W} / \mathrm{m}^{\wedge} 2$. 
Después de obtener los datos de mediciones realizadas durante la implementación del controlador PWM diseñado, la eficiencia se calculó usando (6), y en promedio la eficiencia hallada durante las pruebas de funcionamiento del controlador fue de $71.42 \%$, tomando un total de 56 datos de potencia entregada y recibida, es decir, 224 datos de voltaje y corriente medidos experimentalmente en panel y batería. En la Gráfica 1 se visualiza la eficiencia del controlador con respecto a la radiación solar incidente.

$$
\text { Eficiencia }(\%)=\frac{V B A T * I B A T}{V P A N E L * I P A N E L} * 100 \text { (6) }
$$

\section{Donde:}

VBAT es Voltaje de la batería.

IBAT es Corriente recibida por la batería.

VPANEL es Voltaje del panel.

IPANEL es Corriente entregada por el panel.

Las mediciones experimentales de voltajes, corrientes, potencias y radiación incidente en el panel se realizaron durante 5 horas seguidas, tomando 14 datos de las variables medidas simultáneamente cada hora. Este proceso se repitió por 4 días, obteniendo un total de 280 datos de radiación incidente y de corrientes y voltajes en panel y batería. La radiación solar incidente en el panel variaba durante el tiempo, dependiendo del clima y la nubosidad de la zona [11]. En la Gráfica 1 se puede observar que la eficiencia del controlador PWM disminuye drásticamente cuando la radiación incidente es aproximadamente menor a $280 \mathrm{~W} / \mathrm{m}^{\wedge} 2$, esto se debe a que en estos niveles de radiación el panel produce una corriente de aproximadamente $220 \mathrm{~mA}$ con un voltaje de $15 \mathrm{~V}$ y el controlador PWM toma aproximadamente $120 \mathrm{~mA}$ para su propio funcionamiento, por lo que el porcentaje de potencia que queda para entregar a la batería en el proceso de carga es menor. Para radiaciones mayores a $300 \mathrm{~W} / \mathrm{m}^{\wedge} 2$ el panel genera suficiente potencia de manera que el porcentaje tomado por el controlador para su funcionamiento no representa una pérdida en mayor grado, manteniendo la eficiencia alrededor de $71 \%$.

En cuanto al proceso de descarga controlada de la batería mediante el controlador PWM, se realizaron mediciones de voltaje de batería y corriente entregada por la batería al conectarle una carga de 4W. En la Gráfica 2 se visualiza el comportamiento del voltaje de batería en el transcurso de 320 minutos, y en la Gráfica 3 el comportamiento de la corriente durante el mismo tiempo.
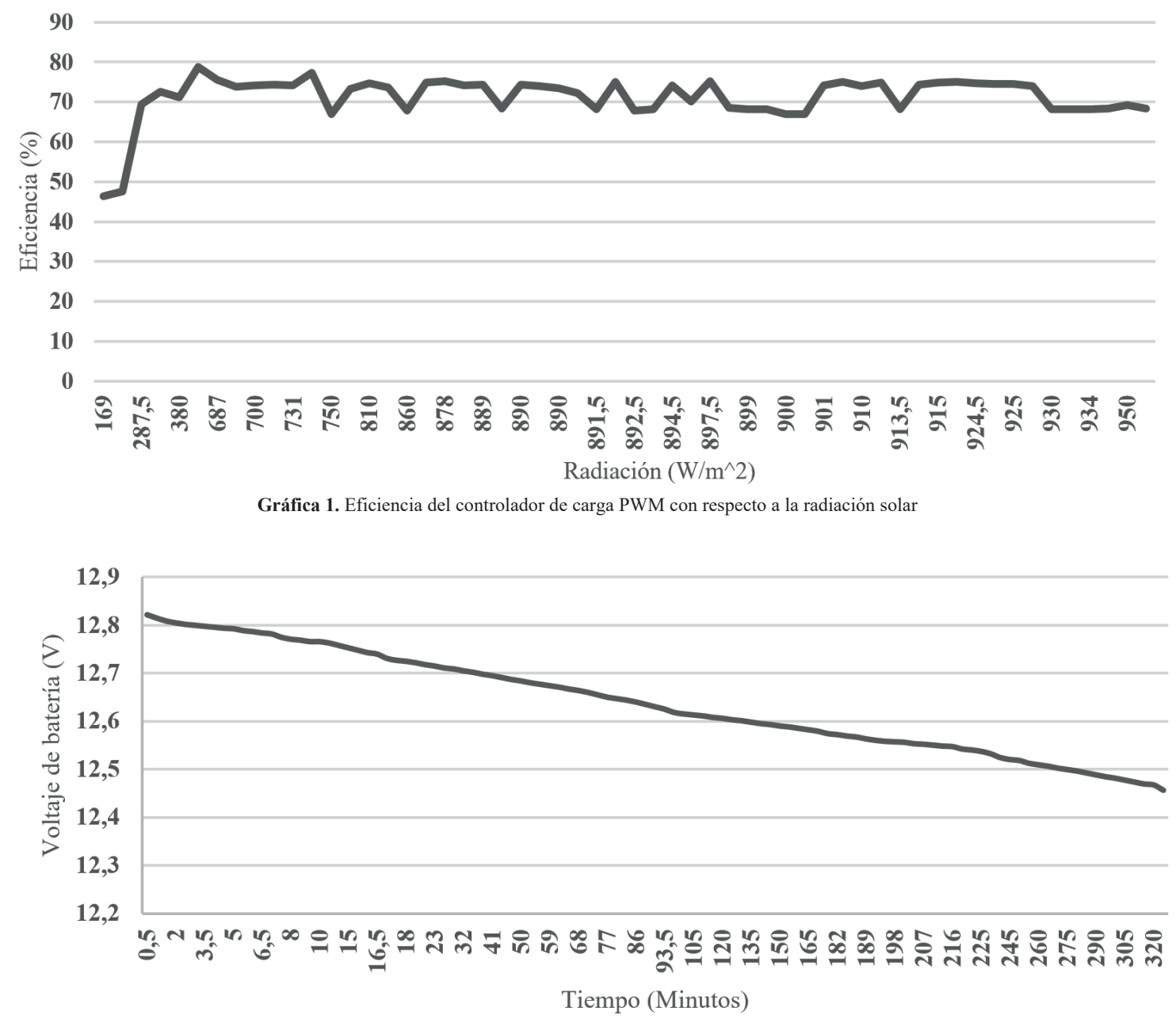

Gráfica 2. Voltaje de batería con respecto al tiempo 


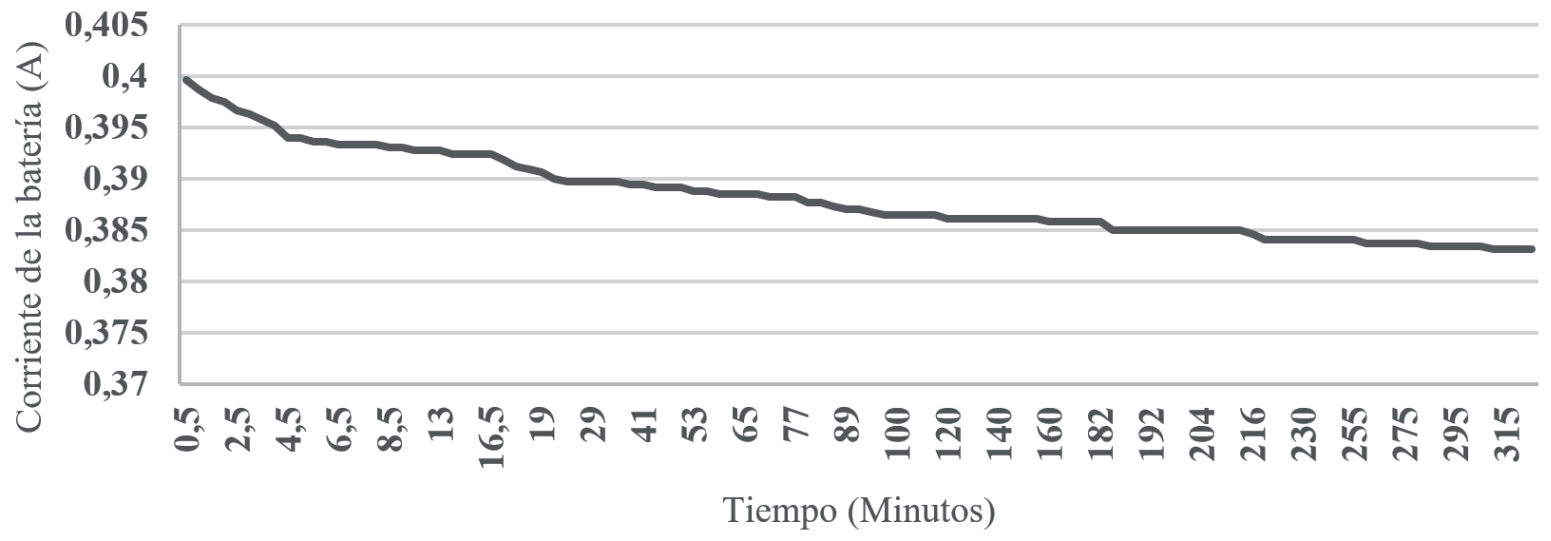

Grafica 3. Corriente de la batería con respecto al tiempo

Según el algoritmo dado por Piller [9], se puede calcular el tiempo en el que la batería se descarga un 90\% utilizando (7) y aplicando un promedio de la corriente que se midió durante las pruebas de descarga implementando el controlador de carga.

$$
\text { Tiempo }(h)=\frac{X * C}{I * 100 \%}(7)
$$

\section{Donde:}

$X$ es Porcentaje de descarga esperado (\%).

$C$ es Capacidad nominal de la batería (Ah).

I es Corriente de consumo de la batería (A).

$$
\text { Tiempo }(h)=\frac{90 \% * 18 A h}{0.388196 A^{*} 100 \%}=41.73 h
$$

Se realizó un análisis de costos para definir el precio comercial del controlador de carga PWM diseñado, tomando como referencia los precios de distribuidores locales que operan en el país en la venta de componentes electrónicos utilizando los precios dados al cliente por las ventas al por mayor, tal como se puede ver en las Tablas I, II y III; además de tener en cuenta los costos indirectos al momento de producir cada uno de los controladores.

Tabla I. Precios unitarios al por mayor de los componentes de la empresa mouser

\begin{tabular}{|c|c|c|c|}
\hline CANTIDAD & 100 & 500 & 1000 \\
\hline COMPONENTES & \multicolumn{2}{|c|}{ PRECIO POR UNIDAD (\$) } \\
\hline MOSFET IRF9540 & 2247 & 1986 & 1566 \\
\hline Regulador 7805 & 1857 & 1641 & 1296 \\
\hline Sensor ACS712 & 12000 & 12000 & 12000 \\
\hline PIC 16f877 & 14040 & 10360,8 & 8172 \\
\hline Capacitores Electrolíticos 47 $\mu \mathrm{F}$ & 130 & 115,12 & 90,8 \\
\hline Capacitores Cerámicos 15pF & 65 & 57,56 & 45,4 \\
\hline Resistencias 1/2 W & 650 & 575,6 & 454 \\
\hline Transistor 2N2222 & 975 & 863,4 & 681 \\
\hline Diodo de potencia 1N5402 & 234 & 207,216 & 125,304 \\
\hline Cristal de 20 MHz & 325 & 287,8 & 227 \\
\hline Conectores banana Hembra & 1950 & 1726,8 & 1362 \\
\hline Conectores banana-Macho & 975 & 863,4 & 681 \\
\hline Diodos Led & 325 & 287,8 & 227 \\
\hline Cable calibre 12 & 1625 & 1439 & 1135 \\
\hline Caja protectora & 10000 & 10000 & 10000 \\
\hline Placa de baquela & 1625 & 1439 & 1135 \\
\hline Disipador & 1950 & 1726 & 1364 \\
\hline Costo total: & 50973 & 45576,496 & 40561,504 \\
\hline
\end{tabular}

Tabla II. Precios unitarios al por mayor de los componentes de la empresa sigma

\begin{tabular}{|c|c|c|}
\hline CANTIDAD & 100 & 500 \\
\hline COMPONENTES & \multicolumn{2}{|c|}{ PRECIO POR UNIDAD (\$) } \\
\hline MOSFET IRF9540 & 6426 & 6360 \\
\hline Regulador 7805 & 506 & 476 \\
\hline Sensor ACS712 & 12500 & 12300 \\
\hline PIC 16f877 & 10729 & 10496 \\
\hline Capacitores Electrolíticos 47 $\mu \mathrm{F}$ & 642 & 572 \\
\hline Capacitores Cerámicos 15pF & 134 & 124 \\
\hline Resistencias 1/2 W & 170 & 140 \\
\hline Transistor 2N2222 & 322 & 318 \\
\hline Diodo de potencia 1N5402 & 303 & 286 \\
\hline Cristal de 20 MHz & 325 & 287,8 \\
\hline Conectores banana Hembra & 1950 & 1726,8 \\
\hline Conectores banana-Macho & 975 & 863,4 \\
\hline Diodos Led & 630 & 595 \\
\hline Cable calibre 12 & 2700 & 2600 \\
\hline Caja protectora & 12000 & 12000 \\
\hline Placa de baquela & 1625 & 1439 \\
\hline Disipador & 963 & 945 \\
\hline Costo total: & 52900 & 51529 \\
\hline
\end{tabular}

Tabla III. Precios unitarios al por mayor de los componentes de la empresa digi-key electronics electronics

\begin{tabular}{|c|c|c|}
\hline CANTIDAD & 100 & 500 \\
\hline COMPONENTES & \multicolumn{2}{|c|}{ PRECIO POR UNIDAD (\$) } \\
\hline MOSFET IRF9540 & 5367 & 4160 \\
\hline Regulador 7805 & 1035 & 813 \\
\hline Sensor ACS712 & 7760 & 6490 \\
\hline PIC 16f877 & 13600 & 13490 \\
\hline Capacitores Electrolíticos 47 $\mu \mathrm{F}$ & 733 & 520 \\
\hline Capacitores Cerámicos 15pF & 414 & 250 \\
\hline Resistencias 1/2 W & 117 & 102 \\
\hline Transistor 2N2222 & 164 & 142 \\
\hline Diodo de potencia 1N5402 & 245 & 231 \\
\hline Cristal de 20 MHz & 360 & 348 \\
\hline Conectores banana Hembra & 5400 & 5160 \\
\hline Conectores banana-Macho & 2700 & 2580 \\
\hline Diodos Led & 1509 & 1498 \\
\hline Cable calibre 12 & 3600 & 3480 \\
\hline Caja protectora & 15000 & 15000 \\
\hline Placa de baquela & 1625 & 1439 \\
\hline Disipador & 2730 & 2670 \\
\hline Costo total: & 62359 & 58373 \\
\hline
\end{tabular}

Las empresas distribuidoras tienen un listado de todos los componentes electrónicos que ofrecen dando a conocer su precio dependiendo de la cantidad pedida ofreciendo un porcentaje de descuento cuando el producto se compra al por mayor. Se tomaron como precios base de componentes los 
valores al por mayor en cada una de las empresas, luego se tomaron como costos indirectos en la fabricación de cada placa un valor de \$5.000, y aplicando un margen de ganancias del 30 $\%$ del producto, dio un total de $\$ 59.300$ como precio comercial con la empresa Mouser Electronics, un precio de $\$ 68.500$ con la empresa Sigma Electrónica, y un precio de $\$ 77.400$ con la empresa Digi-Key Electronics, por lo que se tomó el precio más bajo como el valor comercial del producto.

Tomando como muestra los distribuidores nacionales, en la Tabla IV se realiza una comparativa de los precios comerciales de diferentes controladores de carga PWM con características técnicas similares al diseñado utilizando $12 \mathrm{~V}$ como voltaje nominal de la batería. Se tomó el valor de $70 \%$ de eficiencia para los controladores comerciales debido a que el estado del arte de los controladores de carga PWM especifica que este tipo de tecnología difícilmente supera este porcentaje de eficiencia ya que a diferencia de la tecnología MPPT, la tecnología PWM no realiza una máxima transferencia de potencia, y dado que los proveedores no facilitan el dato de eficiencia en la hoja de características del dispositivo se tomó el valor estándar de eficiencia del $70 \%$.

Tabla IV. Precios de los controladores de carga PWM nacionales electrónica

\begin{tabular}{|c|c|c|c|}
\hline DISTRIBUIDOR & CORRIENTE (A) & VALOR (\$) & EFICIENCIA (\%) \\
\hline Autores & 19 & 59.300 & 71,42 \\
\hline Suneo & 10 & 54.990 & 70 \\
\hline Proviento-SS20L-12 & 20 & 420.000 & 70 \\
\hline Proviento-ECOPULSE & 20 & 309.400 & 70 \\
\hline Proviento-SL20L-12 & 20 & 510.000 & 70 \\
\hline
\end{tabular}

\section{Conclusiones y recomendaciones}

Las mediciones de voltaje y corriente realizadas durante las pruebas experimentales del controlador PWM bajo radiación solar permitieron hallar que la eficiencia promedio del controlador fue de $71.42 \%$, tomando un total de 56 datos de potencia entregada y recibida, es decir 224 datos de voltaje y corriente medidos experimentalmente en panel y batería durante el proceso de carga de la batería, se realizaron las mediciones durante 5 horas seguidas, tomando 14 datos de las variables mediadas simultáneamente cada hora por 4 días.

Durante las pruebas experimentales donde se implementó el controlador de carga PWM diseñado se realizaron mediciones de eficiencia en el proceso de carga y se pudo evidenciar que para radiaciones solares incidentes entre $280 \mathrm{~W} / \mathrm{m}^{\wedge} 2$ y 300 $\mathrm{W} / \mathrm{m}^{\wedge} 2$ la eficiencia decrece hasta un $40 \%$ aproximadamente; para radiaciones solares incidentes menores a $200 \mathrm{~W} / \mathrm{m}^{\wedge} 2$ el panel genera un voltaje menor a $14 \mathrm{~V}$ por lo que el controlador de carga desconecta el panel del circuito dejando la batería cono fuente activa del sistema. Para radicaciones mayores a $300 \mathrm{~W} / \mathrm{m}^{\wedge} 2$ el panel solar produce suficiente potencia para cargar la batería continuamente durante las distintas fases de carga.

La implementación del controlador PWM diseñado en la etapa de descarga de la batería permitió obtener un promedio de corriente de $388 \mathrm{~mA}$ que es entregada por la batería a una carga de $4 \mathrm{~W}$, además de que se pudo observar la disminución del voltaje de la batería durante 320 minutos continuos de descarga. Con la información anterior se calculó que el tiempo de autonomía del sistema fotovoltaico aplicando el controlador de carga PWM diseñado con una carga de $4 \mathrm{~W}$ es de 41.73 horas.

A partir de la Tabla II se pudo comprobar que el objetivo de conseguir un controlador de carga eficiente y a bajo costo se cumple satisfactoriamente, tomando en cuenta que los valores de eficiencia de los controladores de carga PWM de los distribuidores son aproximados a $70 \%$ interpretando la información dada por los distribuidores.

En los sistemas fotovoltaicos autónomos se utilizan comúnmente baterías de plomo ácido de libre mantenimiento ya sea de Gel o de tipo AGM como lo fue en este caso, y el uso de este tipo de baterías obliga a tomar ciertas precauciones al momento de inicializar el controlador de carga PWM, debido a que el voltaje real de circuito abierto debe ser medido tras estar por lo menos tres horas en reposo. Se recomienda esperar este tiempo una vez se desconecta la batería después de pasar por el proceso de carga, de esta manera se garantiza una medición más precisa del voltaje de circuito abierto de la batería por parte del controlador una vez se inicia el algoritmo del estado de carga.

El controlador de carga fue diseñado para un sistema fotovoltaico con un panel de $18 \mathrm{~V}$ y una batería de $12 \mathrm{~V}$ y $18 \mathrm{Ah}$; si se requiere adaptar otro sistema de diferentes especificaciones técnicas, se debe reprogramar en el microcontrolador las variables del voltaje del panel y de voltaje de la batería en cada una de sus estados de carga. Además, si el sistema a adaptar superará los $30 \mathrm{~V}$ de panel o batería, se deben rediseñar los componentes del circuito, en especial el divisor de tensión.

\section{Referencias}

[1] A. Escobar, C. Torres y R. Hincapie, "Conexión de un sistema fotovoltaico a la red eléctrica", Scientia Et Technica, vol. XV, no. 43, pp. 31-36, 2009.

[2] G. E. Francés, "Desarrollo energético sostenible y energías renovables”, Revistas ICE, pp. 1-12, 2012.

[3] S. Singh, "Photovoltaic Water Pumping System for Development of Rural Irrigation", Krishi Sanskriti, vol. 2, no. 5, pp. 3,2015 .

[4] R. B. Thapa, "Off-Grid Energy an Option for Rural Energy Solution”, Imperial Journal of Interdisciplinary Research, vol. 3, pp. 1064-1070, 2017.

[5] D. Hoyos, “Análisis, diseño y construcción de un controlador fotovoltaico", Publicación de Avances en Energías Renovables y Medio Ambiente, vol. 9, pp. 1-6, 2005. 
[6] Y. Yang, Y. Qing, L.Tung, M. Greenleaf y L. Hui, "Integrated Size and Energy Management Design of Battery Storage to Enhance Grid Integration of Large-scale PV Power Plants", IEEE Transactions on industrial electronics, 2017.

[7] J. Chang, H. Liahng y C. Yi Hung, "Solar power generation system for reducing leakage current", International Journal of Electronics, pp. 1-15, 2017.

[8] I. Elzein, "An Evaluation of Photovoltaic Systems MPPT Techniques under the Characteristics of Operational Conditions", Journal of the American Society for Information Science, vol. 2, no. 2, pp. 30-38, 2017.

[9] S. Piller, M. Perrin y A. Jossen, "Methods for state-of-charge determination and their applications", Journal of Power Sources, pp. 113-120, 2001.

[10] G. Sena, R. Marani y A. G. Perri, "Power semiconductors devices for industrial pwm inverters: state of art", International Journal of Advances in Engineering \& Technology, vol. 10, no. 1, pp. 52-65, 2017.

[11] H. Ortiz , C. Escobar y S. Sepúlveda, "Análisis estadístico de variables climatológicas en la ciudad de Cúcuta," Respuestas, vol. 23, no.1, pp. 39-44, 2018.

[12] Suneo: Innovative Energy For Life, "SuneoEnergy", [En línea]. Disponible en: http://suneoenergy.com/index.php/product/epever-10a-pwm-es/. [Accedido en: 02 de diciembre del 2017].

[13] Proviento. S.A.S, "Proviento," [En línea]. Disponible en: http://www.proviento.com.co/controladores.html [Accedido en: 18 de diciembre del 2017]. 